\title{
Ab Initio Study of the Mechanism of Photolytic Deazatization of 2,3-Diazabicyclo[2.2.2]oct-2-ene and 2,3-Diazabicyclo[2.2.1]hept-2-ene
}

\author{
Mark J. Roberson and Jack Simons* \\ Department of Chemistry, University of Utah, Salt Lake City, Utah 84112
}

Received: August 30, 1996; In Final Form: November 6, $1996^{\otimes}$

Computed singlet ground-state and low-lying singlet and triplet excited-state potential energy surfaces of 2,3-diazabicyclo[2.2.2]oct-2-ene (DBO) and 2,3-diazabicyclo[2.2.1] hept-2-ene (DBH) are combined with experimental facts and reasonable models for how excess energy is partitioned among internal and solvent modes to propose mechanisms for the title reactions. In particular, we put forth a mechanism that is consistent with the fact that DBO produces bicyclo[2.2.0]hexane (BCH) and 1,5-hexadiene (HD) in very low quantum yield and in proportions dependent on the spin state of the excited species while DBH produces bicyclo[2.1.0]pentane with near unit efficiency from either singlet or triplet excitation. Our mechanism also rationalizes heavy atom effects on the product ratios in DBO and the stereospecificity and $\mathrm{HD}: \mathrm{BCH}$ yield ratios seen in the products.

\section{Introduction}

Electronic excitation of 2,3-diazabicyclo[2.2.2]oct-2-ene (DBO) (see Figure 1 for structural drawings of all species involved in the DBO and 2,3-diazabicyclo[2.2.1]hept-2-ene (DBH) reactions) induces loss of $\mathrm{N}_{2}$ and production of both 1,5-hexadiene (HD) and bicyclo[2.2.0] hexane (BCH). The HD:BCH product yield ratio depends on the spin state of the excited DBO (we use $\mathrm{DBO}_{\mathrm{S}}$ * to denote singlet excited $\mathrm{DBO}$ and $\mathrm{DBO}_{\mathrm{T}} *$ to denote the triplet), but the total reactive quantum yield seems to be less dependent on spin state and is very small in both cases. Specifically, direct (singlet) excitation of DBO with $360 \mathrm{~nm}$ UV light $(79 \mathrm{kcal} / \mathrm{mol})$ gives 51:49 $\mathrm{HD}: \mathrm{BCH}$ with a total reactive quantum yield $\Phi_{\mathrm{r}}=0.02$ and a fluorescence quantum yield $\Phi_{\mathrm{f}}=0.39$ (the fluorescence lifetime of $\mathrm{DBO}_{\mathrm{S}} *$ is 434 ns). In contrast, triplet excitation using benzophenone sensitization gives 70:30 HD:BCH also with a low reactive quantum yield $\Phi_{\mathrm{r}}=0.014^{1,2}$ but with no phosphorescence. The lifetime of $\mathrm{DBO}_{\mathrm{T}} *$ with respect to all decay processes has recently been estimated $^{2 \mathrm{c}}$ to be $25 \mathrm{~ns}$. The fact that $\mathrm{DBO}_{\mathrm{S}} *$ has time to significantly fluoresce within $434 \mathrm{~ns}$ means that $\mathrm{DBO}_{\mathrm{S}}^{*}$ takes approximately this much time for $61 \%$ of it to surmount any barrier inhibiting movement along its reaction coordinate. Essentially all of the $\mathrm{DBO}_{\mathrm{T}}{ }^{*}$, on the other hand, passes over any barrier on its surface within ca. $25 \mathrm{~ns}$. These data suggest that the initial barrier on the $\mathrm{DBO}_{\mathrm{T}} *$ surface is smaller than that on the $\mathrm{DBO}_{\mathrm{S}} *$ surface.

The structurally similar compound DBH (Figure $1 \mathrm{~b}$ ) reacts with unit efficiency, $\Phi_{\mathrm{r}}=1.00$, from both the singlet and triplet exicted states, producing bicyclo[2.1.0]pentane (BCP). This suggests that the $\mathrm{DBH}_{\mathrm{S}}$ * and $\mathrm{DBH}_{\mathrm{T}}$ * surfaces have smaller initial barriers, as a result of which all initial flux passes promptly onward toward products.

A recent investigation by Anderson and Grissom ${ }^{3}$ has shown that the HD:BCH product ratios are not affected by applied magnetic fields, although the product ratio from singlet DBO* is affected by the presence of heavy atoms. Specifically, in the presence of high concentrations of heavy-atom-containing solvents, the $\mathrm{DBO}_{\mathrm{S}}{ }^{*}$ product yield ratio saturates at the same 70:30 HD:BCH ratio observed in the case of triplet-sensitized $\mathrm{DBO}_{\mathrm{T}}{ }^{*}$, although the singlet's fluoresence quantum yield does not appreciably decrease when the heavy atoms are present (i.e., ca. $39 \%$ still fluoresces).

\footnotetext{
${ }^{\otimes}$ Abstract published in Advance ACS Abstracts, March 1, 1997.
}

Clearly, imporatant questions remain with respect to the above outlined experimental finding on DBO and DBH. (1) What is the fate of $99 \%$ of the triplet and of ca. $59 \%$ of the singlet excited DBO that do not react or emit light? (2) How does one explain the spin-state dependence of the HD:BCH branching ratio? (3) Why is the reactive yield high in $\mathrm{DBH}$ and low in DBO? (4) Do the computed potential energy surfaces agree with the speculations put forth above and thus rationalize why $\mathrm{DBH}$ is different from DBO?

These issues form the focal point of the present study within which we have undertaken an ab initio study of the potential energy surfaces of singlet ground-state DBO and DBH, their lowest-lying excited states, and the reactive pathways leading away from these structures in order to gain insight into the underlying mechanisms. Our findings and one possible interpretation of the experimental facts in terms of them are presented here.

\section{Computational Methods}

As noted earlier, all the chemical species considered in this work are illustrated in Figure 1. Three primary levels of molecular modeling were used to describe the energy profiles along the DBO and DBH reaction paths. Self-consistent field (SCF) molecular orbital and multiconfigurational self-consistent field (MCSCF) reaction energy profiles were obtained using the GAMESS electronic structure program, ${ }^{4}$ and density functional theory (DFT) calculations were performed using the Gaussian 94 program. $^{5}$ In all the ab initio calculations, the 6-31G(d) ${ }^{6}$ atomic orbital basis sets were used for all atoms in all calculations.

As illustrated in recent work from the Schaefer group ${ }^{7}$ on $\mathrm{N}_{2}$ elimination from the methyldiazenyl radical $\mathrm{H}_{3} \mathrm{CNN}\left({ }^{2} \mathrm{~A}^{\prime}\right)$ $\rightarrow \mathrm{H}_{3} \mathrm{C}\left({ }^{2} \mathrm{~A}_{1}\right)+\mathrm{N}_{2}\left(\mathrm{X}^{1} \Sigma_{\mathrm{g}}{ }^{+}\right)$, for basis sets of the quality we have chosen to use here (due to computational practicality), the reaction barrier computed at the SCF level is systematically high by ca. $10 \mathrm{kcal} / \mathrm{mol}$. Employing the same basis but including a high-level treatment of dynamical electron correlation is found to bring the barrier to within $1.3 \mathrm{kcal} / \mathrm{mol}$ of the "best" estimate. A lower level treatment of correlation such as the DFT method empolyed here brings the error from 10 to $5 \mathrm{kcal} / \mathrm{mol}$. The 1.3 $\mathrm{kcal} / \mathrm{mol}$ remaining after high-level correlation tools are used can only be realized by using a much larger atomic orbital basis. Similar trends were found in ref 7 for the computed reaction 
(a)
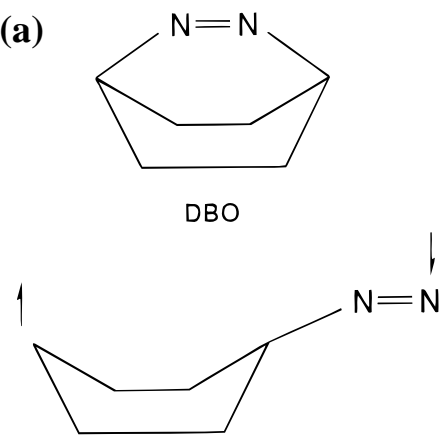

1-Cyclohexyl-4-azenyl (CHA)

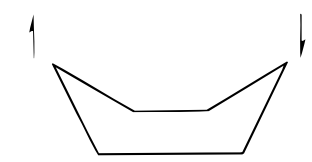

1,4-Cyclohexadiyl (1,4-CHD)

(b)

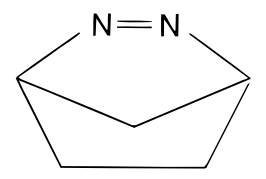

$\mathrm{DBH}$

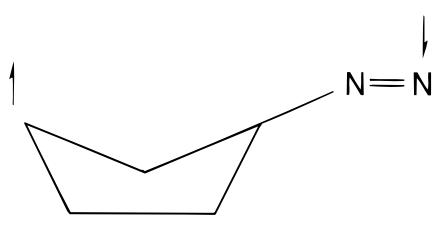

1-Cyclopentyl-3-azenyl (CPA)

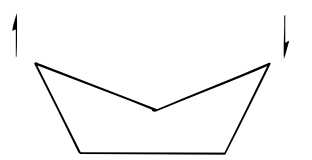

1,3-Cyclopentadiyl (1,3-CPD)

Figure 1. (a) Structures and names of species involved in DBO reactions. (b) Structures and names of species involved in $\mathrm{DBH}$ reactions.

exothermicity $(-27 \mathrm{kcal} / \mathrm{mol}$ for the modest basis at the SCF level; $-20 \mathrm{kcal} / \mathrm{mol}$ when modest correlation is included using the same basis; $-17 \mathrm{kcal} / \mathrm{mol}$ when high-level correlation is treated again with the modest basis; $-18 \mathrm{kcal} / \mathrm{mol}$ when the basis is enhanced and high-level correlation is included). We conclude from this and from our own experience that by using modest correlated methods such as DFT and MCSCF and by employing 6-31G(d) basis sets, our activation energies are likely to be high by ca. $5 \mathrm{kcal} / \mathrm{mol}$ or more, and our exothermicities are expected to be overestimated by similar amounts.

Within the methods discussed above, we employed them as follows. (1) The ground-state singlet $S_{0}$ states for all closedshell species were treated by the restricted Hartree-Fock (RHF) and DFT methods. (2) The generalized valence bond (GVB) method was used for singlet diradical cases. (3) The restricted open-shell Hartree-Fock (ROHF) and DFT methods were used to describe the lowest triplet state $\mathrm{T}_{1}$ at all geometries.

Molecular geometries for the $S_{0}$ and $T_{1}$ states were fully optimized for reactants, intermediates, transition states, and products using the SCF and DFT theories. The SCF-level molecular Hessians were computed to ensure proper curvature of the potential energy surface (PES) at the stationary points,

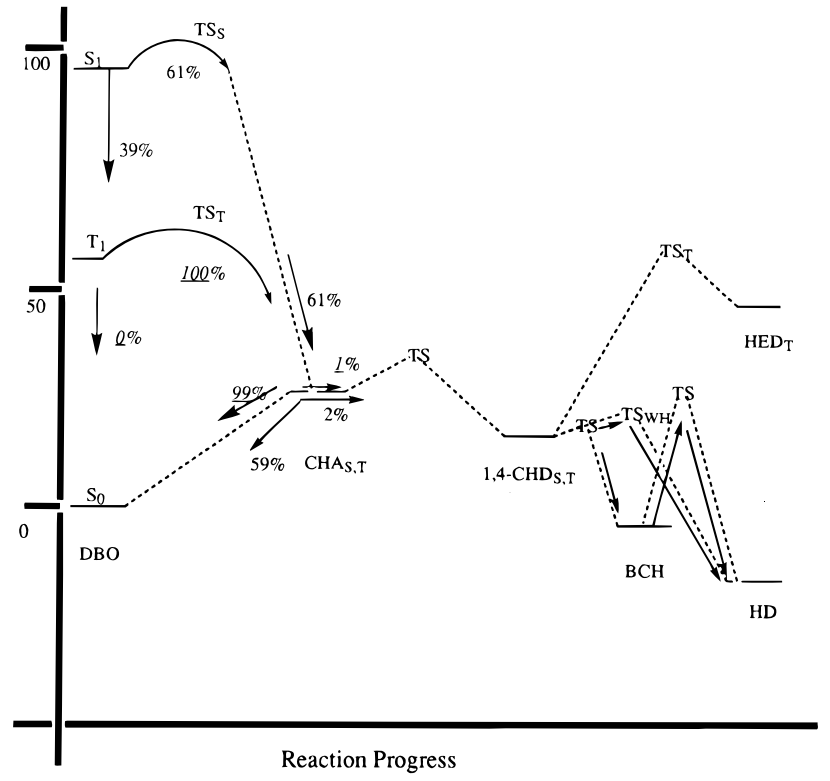

Figure 2. Reaction energy profile for DBO. For the singlet, the initial fluroescence, reclosing, and reaction fractions are noted as percents. The corresponding triplet values are underlined. The subscripts $S$ and T denote singlet and trrplet; the subscript WH denotes a WoodwardHoffmann-allowed path. The energy profile is meant to be only qualitatively correct.

and intrinsic reaction coordinates (IRC) were followed to ensure that the transition states connected the desired local minima. Calculation of the first excited singlet state $S_{1}$, in the neighborhood of the $S_{0}$ ground-state geometry, provided additional challenges. The local minima were indeed found for $\mathrm{S}_{1} \mathrm{DBO}_{\mathrm{S}} *$ and $\mathrm{DBH}_{\mathrm{S}} *$, but the transition states $\mathrm{DBO}_{\mathrm{S}} * \mathrm{TS}$ and $\mathrm{DBH}_{\mathrm{S}} * \mathrm{TS}$ leading away from these minima could not be accurately located. These transition-state energies were therefore estimated by carrying out fully optimized reactive space (FORS) MCSCF calculations at the geometries of the corresponding triplet transition states. Their energies, therefore, are likely to overestimate the respective barriers by amounts exceeding the values given earlier in this section.

\section{Results and Interpretation}

As detailed in the preceding section, the activation energies and exothermicities obtained in our simulations are likely to have significant systematic errors compared to values achieved using much larger atomic orbital basis sets and much higher level treatments of electron correlation. Moreover, nowhere in our simulations are solvation effects included at all. Nevertheless, only (1) the topology (i.e., how the singlet- and tripletstate surfaces of the species shown in Figure 1 are interconnected) of the reactive energy surfaces, (2) the known experimental facts, and (3) reasonable assumptions about the dynamical motions of energized intermediates along the DBO and $\mathrm{DBH}$ reaction paths are used in formulating the reaction mechanisms suggested here. Therefore, we believe that the inadequacies in our quantitative predictions of activation barriers and exothermicities do not invalidate our conclusions, which are about qualitative mechanism rather than quantitative rates of reaction.

A. DBO CN Bond Cleavage To Form CHA. 1. Energy Surface Profiles Early along the Reaction Path. The computed reaction profile for DBO is presented in Figure 2. Our $\mathrm{S}_{0}-\mathrm{S}_{1}$ excitation energy of ca. $85 \mathrm{kcal} / \mathrm{mol}$ compares reasonably well to the $79 \mathrm{kcal} / \mathrm{mol}$ contained in the $360 \mathrm{~nm}$ photons used to excite this state. Other calibrating data are shown in Table 1 where we list the correlated DFT energies for selected com- 
TABLE 1: Correlated Density Functional (DFT) Energies for Selected Species Arising along the DBO and DBH Reaction Paths ${ }^{b}$

\begin{tabular}{|c|c|c|c|}
\hline species & DFT & species & DFT \\
\hline DBO & 0.0 & DBH & 0.0 \\
\hline $\mathrm{DBO}_{\mathrm{T}}{ }^{*}$ & 53 & $\mathrm{DBH}_{\mathrm{T}}{ }^{*}$ & 59 \\
\hline $\mathrm{DBO}_{\mathrm{T}}{ }^{*} \mathrm{TS}$ & 61 & $\mathrm{DBH}_{\mathrm{T}}{ }^{*} \mathrm{TS}$ & 67 \\
\hline $\mathrm{CHA}_{S, \mathrm{~T}}$ & 39 & $\mathrm{CPA}_{S . T}$ & 38 \\
\hline $1,4-\mathrm{CHD}_{\mathrm{S}}+\mathrm{N}_{2}$ & $28^{a}$ & $1,3-\mathrm{CPD}_{\mathrm{S}}+\mathrm{N}_{2}$ & 25 \\
\hline $\mathrm{BCH}+\mathrm{N}_{2}$ & $-5^{a}$ & $\mathrm{BCP}+\mathrm{N}_{2}$ & -7 \\
\hline $\mathrm{HD}+\mathrm{N}_{2}$ & $-13^{a}$ & & \\
\hline $\mathrm{HED}_{\mathrm{T}}+\mathrm{N}_{2}$ & 51 & & \\
\hline
\end{tabular}

${ }^{a}$ In ref 8 , kinetic data is used to infer enthalpy differences (1,4$\mathrm{CHD} \rightarrow \mathrm{BCH}: \Delta H=-35 \mathrm{kcal} / \mathrm{mol}$; $\mathrm{BCH} \rightarrow \mathrm{HD}: \Delta H=-2 \mathrm{kcal} /$ mol). ${ }^{b}$ Energies are in $\mathrm{kcal} / \mathrm{mol}$ relative to $\mathrm{S}_{0}$ reactant species.

pounds arising in the $\mathrm{DBO}$ or $\mathrm{DBH}$ reactions. The energy differences among 1,4-CHD, $\mathrm{BCH}$, and $\mathrm{HD}$ are in reasonable agreement with the enthalpy differences reported by Goldstein and Benzon. ${ }^{8}$ Let us now examine the PESs we computed and consider how they might be used to explain the numerous experimental findings.

From Figure 2 we see the ground-state $S_{0}$ surface evolves smoothly, and without barrier as one $\mathrm{CN} \sigma$ bond is broken, to the intermediate diazenyl CHA (see Figure 1a also), which lies $39 \mathrm{kcal} / \mathrm{mol}$ above the $\mathrm{S}_{0}$ DBO minimum within our approximate calculation that includes no solvent effect.

The first excited triplet state $\mathrm{T}_{1}$ and the first excited singlet state $S_{1}$ must move over barriers, computed to be $<8 \mathrm{kcal} / \mathrm{mol}$ for the triplet and $<11 \mathrm{kcal} / \mathrm{mol}$ for the singlet (both likely significanty overestimated, which we remind by using the < symbol), before also descending to $\mathrm{CHA}$. The fact that significant fluorescence from $S_{1}$ is observed indicates that a barrier does indeed exist in this $\mathrm{DBO}_{\mathrm{S}} *$ case. For both $\mathrm{DBO}_{\mathrm{S}}{ }^{*}$ and $\mathrm{DBO}_{\mathrm{T}}{ }^{*}$, the barriers cannot be very high, since triplet sensitization or $360 \mathrm{~nm}$ photoexcitation provides adequate energy to surmount the respective barriers, since, as noted in the Introduction, $\mathrm{DBO}_{\mathrm{T}} *$ lasts only $25 \mathrm{~ns}$ and $\mathrm{DBO}_{\mathrm{S}} *$ lives for only a fraction of $434 \mathrm{~ns}$ before moving onward along the reaction path.

In the equilibrium structure of CHA, the nitrogen and carbon radicals are separated by about $5 \AA$, so the CHA singlet and triplet states have essentially the same energies at such geometries. It is for this reason that no differentiation is made in Figure 2 among CHA's singlet and triplet states. The chemical consequence of these facts is that hopping between the $S_{0}, S_{1}$, and $T_{1}$ surfaces ought to be reasonably facile in the region of the $\mathrm{CHA}$ structure, and the presence of heavy atoms in the solvent might further facilitate such interconversions and may even cause interconversion at geometries far from the CHA equilibrium.

2. Proposed Evolution of $\mathrm{DBO} \mathrm{O}_{S} *$ and $\mathrm{DBO}_{T} *$ to $\mathrm{CHA}$. Using the 434 ns fluorescence lifetime of $\mathrm{DBO}_{\mathrm{S}} *$ as a "clock" and keeping in mind the $39 \%$ fluorescence yield, we expect that as much as $61 \%$ of the $\mathrm{DBO}_{\mathrm{S}} *$ evolves to a structure similar to the CHA structure within this time scale. Likewise, given the fact that no phosphorescence is observed in the $\mathrm{DBO}_{\mathrm{T}}{ }^{*}$, it is logical to again conclude that nearly $100 \%$ of the $\mathrm{DBO}_{\mathrm{T}} *$ also evolves to the CHA structure within ca. 25 ns. These percentages are shown in Figure 2.

Moreover, during the time ( 25 or 434 ns) it takes to access the CHA geometry, a significant amount of the excess internal energy, which is initially localized in the $-\mathrm{N}=\mathrm{N}-$ moiety by the $\mathrm{n} \rightarrow \pi^{*}$ electronic excitation and evolves into the relative translational movement of the recoiling carbon and nitrogen centers, could be released to the solvent and/or to other internal modes of CHA. However, an amount of energy significantly above equilibrium thermal content must remain in the $\mathrm{CHA}$ to produce even the $1-2 \%$ reaction yields observed because, as discussed in the next section, additional barriers must be overcome to realize the $\mathrm{HD}$ or $\mathrm{BCH}$ products. There is, however, a difference between the singlet and triplet reacting molecules as they approach the DHA geometry. Because $\mathrm{DBO}_{S} *$ has significantly greater excess energy when it surmounts its initial barrier than does $\mathrm{DBO}_{\mathrm{T}}{ }^{*}$, it is likely that $\mathrm{DBO}_{\mathrm{S}} *$ will retain its energy longer and thus be "hotter" in the region of the CHA structure.

B. Reclosing of CHA vs $\mathbf{N}_{2}$ Elimination. 1. Most of the $\mathrm{DBO}_{S}{ }^{*}$ and $\mathrm{DBO}_{T}{ }^{*}$ Flux Recloses. Once the CHA structure is realized, it is possible for the singlet $\mathrm{CHA}$ diradical to reclose to form $\mathrm{S}_{0} \mathrm{DBO}$. This is what we suggest is the fate of $59 \%$ of the $\mathrm{DBO}_{\mathrm{S}}{ }^{*}$ prepared in the photoexcitation case (39\% fluoresces, $59 \%$ recloses, and $2 \%$ eventually reacts). What about the $\mathrm{DBO}_{\mathrm{T}}^{*}$ flux that has overcome its initial barrier and approaches the CHA structure? We suggest that because the singlet- and triplet-state energies are essentially degenerate at the $\mathrm{CHA}$ structure, efficient surface hopping causes rapid interconversions among the spin states. As a result, flux that began on the $\mathrm{DBO}_{\mathrm{T}} *$ surface can, after spin-state conversion, undergo the same closing step outlined above for $\mathrm{DBO}_{\mathrm{S}} *$. Thus, we suggest that $99 \%$ of the $\mathrm{DBO}_{\mathrm{T}}{ }^{*}$ generated via triplet sensitization $(1 \%$ reacts, $0 \%$ phosphoresces) also recloses to form $\mathrm{S}_{0} \mathrm{DBO}$. Because DBO contains a six-membered hydorcarbon ring that can undergo facile boat-to-chair interconversion, especially given its greater-than-thermal energy content, such reclosure is to be expected (this is not the case for DBH, whose five-membered ring's lack of flexibility is expected to greatly inhibit reclosure).

2. Small Percent of Flux that Reacts Proceeds Differently for $\mathrm{DBO}_{S} *$ and $\mathrm{DBO}_{T} *$. For the $1-2 \%$ of $\mathrm{DBO}^{*}$ that reaches CHA and does not reclose but moves on to products, something different happens. On the lowest singlet or triplet surfaces, passage over a $<12 \mathrm{kcal} / \mathrm{mol}$ barrier leads from $\mathrm{CHA}$ to elimination of singlet closed-shell $\mathrm{N}_{2}$ (the lowest triplet state of $\mathrm{N}_{2}$ is much higher in energy) and production of singlet or triplet 1,4-CHD. For any HD of BCH product to subsequently be formed, the $\mathrm{CHA}$ reacting species must retain at least enough internal energy to surmount this barrier.

a. Singlet Case. Once formed, the 1,4-CHD diradical has several exit channels in its singlet state. A WoodwardHoffmann-allowed $C_{2}$ transition state leads to final product HD molecules, with a barrier height $<7 \mathrm{kcal} / \mathrm{mol}$ above the diradical. The singlet diradical can alternatively pass over the lower $<2$ $\mathrm{kcal} / \mathrm{mol}$ barrier to form the other final product $\mathrm{BCH}$ from which it can evolve over another Woodward-Hoffmann-forbidden $C_{1}$ (but very nearly $C_{s}$ ) barrier, $<21 \mathrm{kcal} / \mathrm{mol}$ in height, to rearrange to HD. It makes sense that some of the flux of CHA that has enough excess energy to surmount the $<12 \mathrm{kcal} / \mathrm{mol}$ barrier to produce 1,4-CHD could retain enough energy to also surmount the subsequent $<2,<7$, and $<21 \mathrm{kcal} / \mathrm{mol}$ barriers (see Figure 2 ). Note that within this interpretation of how singlet DBO reacts, the HD product molecules can be formed in either or both of two manners: directly from the 1,4-CHD diradical or by rearrangement of $\mathrm{BCH}$, the former proceeding via a Woodward-Hoffmann-allowed path and the latter via a nonallowed path. These two paths are shown by arrows in Figure 2 .

b. Triplet Case. The triplet diradical 1,4-CHD has but one exit channel once singlet $\mathrm{N}_{2}$ eliminates, passing over a high $<49 \mathrm{kcal} / \mathrm{mol}$ barrier to form triplet hex-1-ene-5,6-diyl (HED), which must then relax to its singlet spin state before it can close to form HD. Because surmounting the $<49 \mathrm{kcal} / \mathrm{mol}$ barrier would require significanlty more internal energy than even that needed by CHA to overcome its $<12 \mathrm{kcal} / \mathrm{mol}$ barrier to $1,4-$ CHD, we consider this path to be chemically irrelevant. It is most likely that triplet $1,4-\mathrm{CHD}$ continues to undergo spin-state 


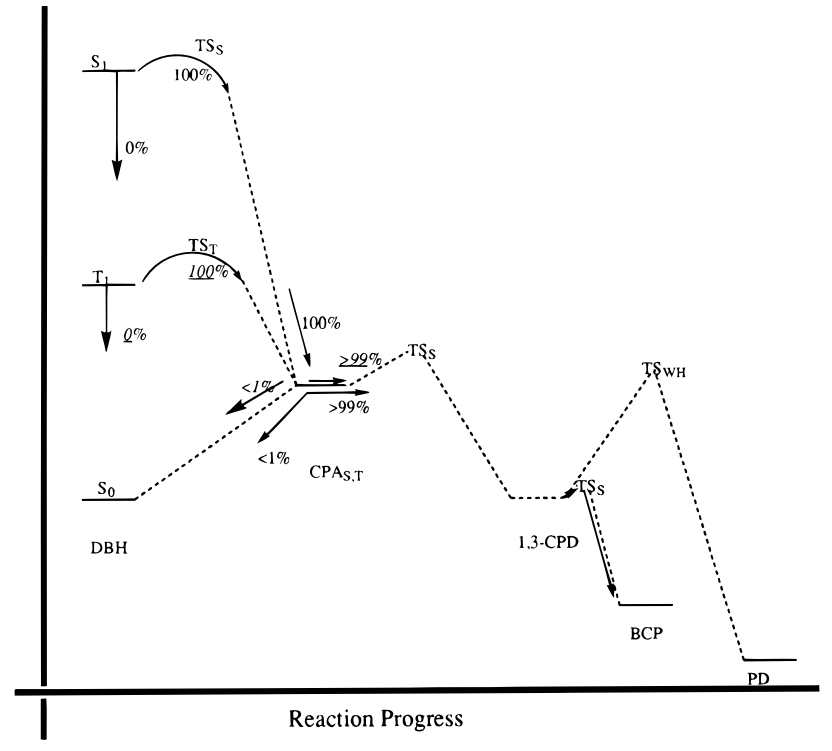

Figure 3. Reaction energy profile for DBH. For the singlet, the initial fluroescence, reclosing, and reaction fractions are noted as percents. The corresponding triplet values are underlined. The subscripts S and T denote singlet and trrplet; the subscript WH denotes a WoodwardHoffmann-allowed path. The energy profile is meant to be only qualitatively correct.

interconversion, producing singlet 1,4-CHD whose fate is detailed in the preceding paragraph.

C. What Is Different about the DBH Molecule? 1 . Passing over the First Barriers on $\mathrm{DBH}_{T}{ }^{*}$ and $\mathrm{DBH}_{S}{ }^{*}$. The reaction coordinate diagram for $\mathrm{DBH}$ is presented in Figure 3. As with $\mathrm{DBO}$, the ground-state surface leads, without a barrier, to the carbylazenyl CPA whose energy is $38 \mathrm{kcal} / \mathrm{mol}$ above the $\mathrm{DBH} \mathrm{S}_{0}$ minimum. The barriers on the $\mathrm{DBH} \mathrm{T}_{1}$ and $\mathrm{S}_{1}$ states leading to CPA are $<8 \mathrm{kcal} / \mathrm{mol}$ and $<12 \mathrm{kcal} / \mathrm{mol}$, respectively, although again the height of the $S_{1}$ barrier is probably more overestimated than is the triplet barrier. In both cases, the barriers must, in reality, be small because the reactive yield for DBH is near unity. Once CPA is accessed, singlettriplet interconversion can be facile because here the surfaces are nearly isoenergetic. To this point, our suggestions for DBH are very nearly identical with those for DBO, except for the latter $39 \%$ of the sinlget flux that is lost to fluorescence. However, from this point on, the major difference appears.

2. Reclosure Is Very Improbable for CPA. The fact that the total product quantum yield for bicyclo[2.1.0]pentane (BCP) is near unity indicates that reclosure of CPA to reform DBH is much rarer than passage of CPA over subseqent barriers to products. The high reactive quantum yield and low reclosure rate are in marked contrast to the case for DBO. It is our belief that the much more rigid five-membered hydrocarbon ring in $\mathrm{DBH}$ causes reclosure of CPA to DBH to occur much less often than reclosure of the six-membered ring in CHA. It is not because there exists an energy barrier to reclosure in CPA but is more likely a result of how the excess energy is distributed among the internal modes of CPA. That is, although the nascent CPA may retain a considerable amount of the excess energy it achieves from passing over the $S_{1}$ or $T_{1}$ barrier, it is unlikely to retain enough of this energy in its very "stiff" ring-puckering mode to permit reclosure. In contrast, even with a similar total amount of internal energy, CHA is more likely to significantly populate the much softer ring-distortion modes present in its six-membered ring. This seems to explain one of the primary differences between $\mathrm{DBO}$ and $\mathrm{DBH}$ - the low reactive quantum yield in the former and near unit quantum yield in the latter.

3. From CPA Onward to Products. Once CPA is formed, passage over a $<10 \mathrm{kcal} / \mathrm{mol}$ barrier leads to loss of ground- state singlet $\mathrm{N}_{2}$ and formation of singlet or triplet 1,3-CPD diradical. The singlet population of this diradical can then close to form the final products BCP as shown by the arrows in Figure 3. Figure 3 shows that another exit channel leading over a $<38$ $\mathrm{kcal} / \mathrm{mol}$ barrier to generate 1,4-pentadiene (PD) also exists, but this product has not been seen experimentally (likely because the latter barrier is too high to access because this late along the reaction coordinate, the reacting molecules possess inadequate internal energy).

D. Stereoisomer Distribution in DBO Productts. 1 . Bicyclohexane Isomers. a. Puzzling Facts. Starting from triplet-excited $\mathrm{DBO}_{\mathrm{T}}{ }^{*}$, the stereochemistry of the $\mathrm{BCH}$ product is found to be 58:42 exo:endo, where exo signifies inversion of configuration at the bridging carbon center. The deviation from a 50:50 ratio expected from racemization is consistent with a kinetic isotope effect. These data and our earlier arguments concerning the fate of $\mathrm{DBO}_{\mathrm{T}} *$ flux are consistent with the proposal that reaction proceeds through $\mathrm{CHA}$ and thence 1,4CHD whose spin states are rapidly interconverting, since $\mathrm{BCH}$ can only be formed by closure of singlet 1,4-CHD.

Starting from singlet-excited $\mathrm{DBO}_{\mathrm{S}}{ }^{*}$, the stereochemistry of the product $\mathrm{BCH}$ is 83:17 exo:endo, not 58:42 as in the triplet case. However, when the singlet $\mathrm{DBO}_{\mathrm{S}} *$ reaction is carried out in the presence of heavy-atom-containing solvent, the 58:42 exo: endo ratio is again achieved.

Analogous puzzles occur when the HD:BCD product ratios arising in the triplet and singlet cases are examined. When $\mathrm{DBO}_{\mathrm{T}}^{*}$ is the precursor, $\mathrm{HD}: \mathrm{BCH}=70: 30$ is observed, but when $\mathrm{DBO}_{\mathrm{S}} *$ is employed, $\mathrm{HD}: \mathrm{BCH}=51: 49$ is found. In addition, when $\mathrm{DBO}_{\mathrm{S}} *$ is used and the reaction is carried out in a heavy-atom-containing solvent, the $\mathrm{HD}: \mathrm{BCH}=70: 30$ product ratio is again achieved.

Both the exo:endo and $\mathrm{HD}: \mathrm{BCH}$ ratios displayed by $\mathrm{DBO}_{\mathrm{S}}$ * differ from those of $\mathrm{DBO}_{\mathrm{T}}{ }^{*}$, except when heavy atoms are present, in which case the $\mathrm{DBO}_{S} *$ reaction gives the same (58: 42 and 70:30) ratios, respectively, as found for $\mathrm{DBO}_{\mathrm{T}}{ }^{*}$.

b. Our Interpretation. i. DBO ${ }_{S}^{*}$ Case. How do we explain these facts? We believe that in the singlet case in the absence of heavy atom effects, a fraction $X$ of the nascent and eventuallyto-react $\mathrm{DBO}_{\mathrm{S}}{ }^{*}$, on its way to forming singlet $\mathrm{CHA}$, promptly undergoes back-side displacement ${ }^{2 \mathrm{~b}}$ of the carbon radical center on the carbon atom bound to the $\mathrm{NN}$ moiety, ejecting $\mathrm{N}_{2}$ and producing exo-BCH with $100 \%$ inversion of configuration. Not all of the $\mathrm{DBO}_{\mathrm{S}} *$ have just the right recoil momentum to undergo this displacement reaction. The remaining flux proceeds onward to CHA, most of which then reclosed to reform $S_{0}$ DBO. However, of the singlet $\mathrm{CHA}$ that does not reclose, another fraction $Y$ goes through the 1,4-CHD diradical to also form $\mathrm{BCH}$ but in the same 58:42 exo:endo ratio observed in the triplet case. The remaining fraction $Z$ goes through the same 1,4CHD to produce HD.

There exist three relationships among the above three fractions of reactive singlet species that started as $\mathrm{DBO}_{\mathrm{S}} *(X, Y$, and $Z)$ that can be expressed in terms of the following simultaneous equations:

$$
\begin{gathered}
\text { mass conservation } X+Y+Z=1.0 \\
\text { BCH exo:endo ratio }(X+0.58 Y) /(X+Y)=0.83 \\
\text { BCH:HD product ratio }(X+Y) / Z=49 / 51
\end{gathered}
$$

Solving these three equations in three unknowns yields $X=$ $0.292, Y=0.198, Z=0.510$. This means that of the $2 \%$ of $\mathrm{DBO}_{\mathrm{S}} *$ that neither fluoresces nor recloses, $29 \%$ undergoes prompt formation of exo-BCH via back-side displacement; the remaining $71 \%$ goes on to $\mathrm{CHA}$. From there, $20 \%$ goes on to form endo-CHA and $51 \%$ produces HD. 
TABLE 2: Product Distributions from Deuterium-Labeled $\mathrm{DBO}^{a}$

\begin{tabular}{|c|c|c|}
\hline & direct photolysis (\%) & triplet sensitized $(\%)$ \\
\hline \multicolumn{3}{|c|}{ BCH Stereochemistry } \\
\hline exo-BCH & 83 & 58 \\
\hline endo-BCH & 17 & 42 \\
\hline \multicolumn{3}{|c|}{ Hexadiene Stereochemistry } \\
\hline$E, E-\mathrm{HD}$ & 13 & 15 \\
\hline Z,Z-HD & 4 & 5 \\
\hline$E, Z$-HD & 83 & 80 \\
\hline
\end{tabular}

${ }^{a}$ Data from Edmunds and Samuel. J. Chem. Soc., Perkin Trans. 1 1989, 1267.

ii. $\mathrm{DBO}_{T}{ }^{*}$ Case. Using this same reaction scheme, we can also explain the product ratios seen when $\mathrm{DBO}_{\mathrm{T}} *$ is the precursor. In this triplet $\mathrm{DBO}_{\mathrm{T}} *$ case, we speculate that the same things happen except that none of the nascent $\mathrm{DBO}^{*}$, on its way to forming triplet $\mathrm{CHA}$, can undergo the prompt backside configuration inverting step because neither triplet $\mathrm{N}_{2}$ nor triplet $\mathrm{BCH}$ is energetically accessible. Thus, all the reacting flux of triplet $\mathrm{DBO}_{\mathrm{T}}{ }^{*}$ must continue on to $\mathrm{CHA}$ and subsequently to 1,4-CHD where spin-state interconversion to the singlet is facile. If our model is correct, we can now predict the $\mathrm{BCH}: \mathrm{HD}$ ratio expected for triplet $\mathrm{DBO}_{\mathrm{T}} *$ using the $Y$ and $Z$ values computed above for the singlet and simply ignoring (i.e., setting $X=0$ ) the back-side attack fraction. So doing, we predict $\mathrm{BCH}: \mathrm{HD}=Y: Z=0.198: 0.510=28: 72$, nearly the 30:70 ratio actually observed for triplet DBO*. For the exo: endo ratio, we find 0.58 , as expected.

iii. $\mathrm{DBO}_{S}{ }^{*}$ Case When Heavy Atoms or Magnetic Fields Are Present. This same kinetic scheme can be used to rationalize the observations when the $\mathrm{DBO}_{\mathrm{S}} *$ reaction is carried out with heavy-atom-containing solvents. We recall that the $\mathrm{DBO}_{\mathrm{S}}{ }^{*}$ fluoresence quantum yield is not significanlty altered when $\mathrm{DBO}_{\mathrm{S}} *$ reacts in solvents containing heavy atoms. This implies that singlet-to-triplet interconversion is not taking place, at least within $434 \mathrm{~ns}$, in the $\mathrm{n} \pi^{*}$ excited $\mathrm{DBO}_{\mathrm{S}}{ }^{*}$ prior to rupture of the first $\mathrm{CN}$ bond.

Once the first $\mathrm{CN}$ bond is fully or mostly broken and $\mathrm{DBO}_{\mathrm{S}}$ * is on its way to CHA, the spacing between the singlet and triplet energy surfaces becomes smaller, as a result of which heavyatom-induced spin interconversion is more likely to occur. Most likely, the effect will be to generate singlet-triplet interconversion after the first $\mathrm{CN}$ bond in $\mathrm{DBO}_{\mathrm{S}} *$ has fragmented and is continuing all the way to the CHA geometry. By doing so, the heavy atom can act to interrupt the prompt back-side attack of the carbon radical center on the carbon atom bound to the $\mathrm{NN}$ group, whose elimination to give $\mathrm{N}_{2}$ and $\mathrm{BCH}$ can only occur if flux remains on the singlet surface. This singlet-totriplet surface hop is what we believe causes the $\mathrm{DBO}_{\mathrm{S}} *$ to behave like $\mathrm{DBO}_{\mathrm{T}} *$ in such cases. What is not clear, however, is why applied magnetic fields do not affect the singlet DBO* product ratio as much as heavy-atom-containing solvents do. It may simply be that the perturbation induced by laboratoryreasonable magnetic fields cannot cause singlet-to-triplet interconversion soon enough after rupture of the first $\mathrm{CN}$ bond to interrupt the back-side attack pathway. Further exploration of this question is needed.

2. Hexadiene Isomers. As summarized in Table 2, both singlet and triplet DBO* form appreciable (ca. 20\%) and similar amounts of the Woodward-Hoffmann-forbidden isomers $E, E$ $\mathrm{HD}$ and $Z, Z-\mathrm{HD},{ }^{9}$ formed presumably by $1,4-\mathrm{CHD}$ closing to $\mathrm{BCH}$, which subsequently rearranges (see Figure 2) to HD. In both spin cases, the Woodward-Hoffmann-allowed E,Z-HD isomer is formed in ca. $80 \%$ abundance. The lack of spin-state dependence of this product yield suggests that HD is formed from the same diradical precursor in the singlet and triplet $\mathrm{DBO}^{*}$ cases, which is what our model postulates.

It should be noted that even $20 \%$ of the forbidden product is far in excess of the proportion expected from a system near thermal equilibrium given that the forbidden barrier lies $<14$ $\mathrm{kcal} / \mathrm{mol}$ higher in energy than the allowed barrier. This provides further evidence that the reacting species have retained substantial internal energy throughout the reaction process and have not reached thermal equilibrium along the reaction paths.

\section{Summary}

We have given a detailed rationalization of the various experimental findings surrounding singlet or triplet excitation of DBO and DBH. This rationalization is based on computed potential energy surfaces, including reactants, products, intermediates, and transition states, for the $\mathrm{S}_{0}, \mathrm{~S}_{1}$, and $\mathrm{T}_{1}$ states. Differences between DBO and DBH photolysis quantum yields, stereoisomer product yields in DBO, and heavy atom effects are all accounted for in the mechanism developed here. The absence of a magnetic field effect in DBO's reactivity is not yet fully understood, although we speculate on its origin.

The essential aspects of our proposed mechanism are the following. (1) $\mathrm{DBO}_{\mathrm{S}} *$ and $\mathrm{DBO}_{\mathrm{T}} *$ proceed to $\mathrm{CHA}$ where efficient spin-state interconversion occurs. Reclosure of CHA to $\mathrm{S}_{0} \mathrm{DBO}$ is then facile because of the flexible six-membered ring in CHA. (2) $\mathrm{DBH}_{\mathrm{S}} *$ and $\mathrm{DBH}_{\mathrm{T}}$ * analogously proceed to $\mathrm{CPA}$, but reclosure is rare because the five-membered ring in $\mathrm{CPA}$ is too stiff to permit it. (3) $\mathrm{DBO}_{\mathrm{S}} *$ and $\mathrm{DBO}_{\mathrm{T}}{ }^{*}$ flux passing through $\mathrm{CHA}$ moves to $1,4-\mathrm{CHD}$ where branching to $\mathrm{BCH}$ and CHD occurs. (4) For the more energetic $\mathrm{DBO}_{\mathrm{S}} *$, prompt back-side elimination of $\mathrm{N}_{2}$ can also take place early along the reaction coordinate to produce additional $\mathrm{BCH}$. (5) In the presence of heavy atoms, the above prompt back-side displacement is interrupted by $\mathrm{S}_{1} \rightarrow \mathrm{T}_{1}$ interconversion, thus causing $\mathrm{DBO}_{S} *$ in the presence of heavy atoms to behave like $\mathrm{DBO}_{\mathrm{T}}^{*}$.

Acknowledgment. The authors thank Mr. Mark Anderson for his invaluable assistance in framing the issues discussed in this paper and the National Science Foundation for financial support through Grant No. 9116286.

\section{References and Notes}

(1) (a) Engel, P. S.; Hayes, R. A.; Keifer, L.; Szilagyi, S.; Timberlake, J. W. J. Am. Chem. Soc. 1978, 100, 1876. (b) Engel, P. S.; Horsey, D. W.; Keys, D. E.; Nalepa, C. J.; Soltero, L. R. J. Am. Chem. Soc. 1983, 105, 7108.

(2) (a) Clark, W. D. K.; Steel, C. J. Am. Chem. Soc. 1971, 93, 6347. (b) Roth, W. R.; Martin, M. Tetrahedron Lett. 1967, 4695. (c) We thank one of our reviewers for informing us that R. Caldwell recently determined this lifetime.

(3) Anderson, M. A.; Grissom, C. B. J. Am. Chem. Soc. 1995, 117, 5041.

(4) Schmidt, M. W.; Baldridge, K. K.; Boatz, J. A.; Elbert, S. T.; Gordon, M. S.; Jensen, J. H.; Koseki, S.; Matsunaga, N.; Nguyen, K. A.; Su, L. J.; Windus, T. L.; Dupuis, M.; Montgomery, J. A. J. Comput. Chem. 1993, 14, 1347.

(5) Frisch, M. J.; Trucks, G. W.; Schlegel, H. B.; Gill, P. M. W.; Johnson, B. G.; Robb, M. A.; Cheeseman, J. R.; Keith, T.; Petersson, G. A.; Montgomery, J. A.; Raghavachari, K.; AL-Laham, M. A.; Zakrzewski, V. G.; Ortiz, J. V.; Foresman, J. B.; Cioslowski, J.; Stefanov, B. B.; Nanayakkara, A.; Challacombe, M.; Peng, C. Y.; Ayala, P. Y.; Chen, W.; Wong, M. W.; Andres, J. L.; Replogle, E. S.; Gomperts, R.; Martin, R. L.; Fox, D. J.; Binkley, J. S.; Defrees, D. J.; Baker, J.; Stewart, J. P.; HeadGordon, M.; Gonzalez, C.; Pople, J. A. Gaussian 94, Revision B.1; Gaussian, Inc.: Pittsburgh, PA, 1995.

(6) Ditchfield, R.; Hehre, W. J.; Pople, J. A. J. Chem. Phys. 1972, 56 , 2257.

(7) Wu, C. H.; Schaefer, H. F. J. Chem. Phys. 1994, 101, 1289.

(8) Goldstein, M. J.; Benzon, M. S. J. Am. Chem. Soc. 1972, 94, 5119.

(9) Edmunds, A. J. F.; Samuel, C. J. J. Chem. Soc., Perkin Trans. 1 1989, 1267. 